\title{
London superconductor and time-varying mesoscopic LC circuits
}

\author{
I. A. Pedrosa and L. Nascimento \\ Departamento de Física, CCEN, Universidade Federal da Paraíba, \\ Caixa Postal 5008, 58059-900, João Pessoa, PB, Brazil. \\ *e-mail: iapedrosa@fisica.ufph.br, luciano.fisicacex@gmail.com
}

Received 24 February 2021; accepted 9 April 2021

In this work, we study the classical and quantum dynamics of a London superconductor and of a time-dependent mesoscopic or nanoscale LC circuit by assuming that the inductance and capacitance vary exponentially with time at a constant rate. Surprisingly, we find that the behavior of these two systems is equivalent, both classically and quantum mechanically, and can be mapped into a standard damped harmonic oscillator which is described by the Caldirola-Kanai Hamiltonian. With the aid of the dynamical invariant method and Fock states, we solve the time-dependent Schrödinger equation associated with this Hamiltonian and calculate some important physical properties of these systems such as expectation values of the charge and magnetic flux, their variances and the respective uncertainty principle.

Keywords: London superconductor; LC circuit; london equations; invariant method; Schrödinger equation; coherent states.

DOI: https://doi.org/10.31349/RevMexFis.67.050501

\section{Introduction}

Over the past years, the study of classical and/or quantum systems that may be described by the same mathematical formalism has received considerable attention of physicists and continues to be a living and interesting research area of modern physics. This interest is because the elucidation of similarities in the behavior of systems studied by different areas of physics frequently allows the knowledge of each area to help the understanding of the other.

In recent years, the phenomenon of superconductivity has been the focus of considerable theoretical and experimental study in the literature. The origin of this great interest is due to its potential application in various branches of physics [1-6]. This phenomenon was first observed in Leiden by Onnes in 1911 [7] when he noted that the resistance of a rod of frozen mercury suddenly drops to zero when cooled to the boiling point of helium, $4.2 \mathrm{~K}$, and in 1933 [8], Meissner and Ochsenfeld discovered that this phenomenon expels the magnetic field from within the superconductor. This discovery is nowadays known as the Meissner-Ochsenfeld effect. Later, other important contributions to the study of superconducting materials were given by some researchers [9-13]. However, a great deal of our knowledge on superconductivity can be obtained from the London equations $[9,13]$.

With the development of nanometer techniques and microelectronics, classical and quantum effects of mesoscopic or nanoscale circuits have attracted a lot of interest from physicists [14-20]. In the study of mesoscopic circuits, an LC (inductance L and capacitance $\mathrm{C}$ ) circuit represents a fundamental cell. To our knowledge, the quantization of this circuit was first performed by Louisell in the 1970s [21]. This author has studied the quantum effects of a nondissipative mesoscopic LC circuit with a source and expressed its fluctuations in the vacuum state. Based on Louisell's work and with the progress of quantum information and quantum computa- tion, many articles concerned with mesoscopic or nanoscale circuits have been published in the literature [1-5, 22-25].

The connection of superconductors with a mesoscopic LC circuit can be made through the Josephson junctions. In fact, one can consider Josephson junctions connected to an inductance by a capacitor $[1,2,6]$. So, it is of high interest to academics to study mesoscopic LC circuits from both classical and quantum viewpoints. Yet, in recent years physicists have found that a practical solid quantum computer might be built by using some solid-state devices, for example, taking a Josephson junction as its core $[1,2]$. Here, it is worth remembering that a Josephson junction is composed of two superconductors weakly connected by a thin layer of insulating material $[1,2,6]$.

In the present paper, stimulated by a connection between superconductors and mesoscopic LC circuits, we investigate the behavior of a London superconductor and a mesoscopic LC circuit with a time-varying inductance increasing exponentially and a time-dependent capacitance decreasing exponentially from the classical and quantum viewpoints. Notably, we find that the behavior of these systems is identical and can be described by the Caldirola-Kanai Hamiltonian. Furthermore, using the dynamical invariant method developed by Lewis and Riesenfeld [26] and Fock states, we easily solve the time-dependent Schrödinger associated with this Hamiltonian and write its solutions in terms of a special solution of the Milne-Pinney equation [27, 28]. Finally, we use Fock states to calculate some quantum properties of these systems, such as the expectation values of the charge and magnetic flux, their quantum variances, and the corresponding Heisenberg uncertainty principle.

This paper is organized as follows. In Sec. 2, we discuss the classical equivalence of the London superconductor and of the time-dependent mesoscopic LC circuit. The quantum equivalence is presented in Sec. 3. In Sec. 4, we conclude the paper with a short summary. 


\section{Classical description}

\subsection{London Superconductor}

In this subsection, we discuss the classical behavior of the London superconductor. In order to do so, let us first consider, for the sake of clarity on the exposition of the mathematical formalism, the classical Maxwell's equations with free charge and currents sources which can be written in the form

$$
\begin{aligned}
\nabla \cdot \vec{D} & =\rho, \\
\nabla \times \vec{E} & =-\frac{\partial \vec{B}}{\partial t}, \\
\nabla \cdot \vec{B} & =0, \\
\nabla \times \vec{H} & =\frac{\partial \vec{D}}{\partial t}+\vec{J} .
\end{aligned}
$$

where $\rho$ is the total density charge. The constitutive equations relating the fields are given by $\vec{D}=\varepsilon \vec{E}$ and $\vec{B}=\mu \vec{H}$ where $\varepsilon$ and $\mu$ are, respectively, the electric permittivity and the magnetic permeability. Strictly speaking, the electric permittivity and the magnetic permeability are complex, but in this paper, we assume that they are real. Further, the charge and current satisfy the continuity equation

$$
\nabla \cdot \vec{J}=-\frac{\partial \rho}{\partial t}
$$

where $\vec{J}$ represent the total current density.

The electromagnetic equations of the superconductor, that is, the London's equations, are given by [9, 13, 29]

$$
\begin{gathered}
\frac{\partial \vec{J}_{s}}{\partial t}=\frac{1}{\mu \lambda_{L}^{2}} \vec{E}, \\
\nabla \times \vec{J}_{s}=-\frac{n_{s} e_{s}^{2}}{m_{s}} \vec{B},
\end{gathered}
$$

where

$$
\lambda_{L}^{2}=\frac{m_{s}}{\mu n_{s} e_{s}^{2}},
$$

is the London penetration depth. In the above expressions, $\overrightarrow{J_{s}}$ is the supercurrent density, and $n_{s}, m_{s}$ and $e_{s}$ are, respectively, the density of electrons in a superconducting state, the mass, and the charge of the superconducting electrons. Now, the total current density is

$$
\vec{J}=\overrightarrow{J_{n}}+\overrightarrow{J_{s}}
$$

where $\overrightarrow{J_{n}}$ is the current density associated with the normal electrons given by

$$
\overrightarrow{J_{n}}=\sigma \vec{E}
$$

with $\sigma$ being the electric conductivity. Then, differentiating Eq. (9) with respect to time and using the Maxwell and London equations as well as the continuity equation (5), we obtain the equation of motion for the total charge within a certain volume of the superconductor as

$$
\ddot{q}(t)+\frac{\sigma}{\varepsilon} \dot{q}(t)+\omega^{2} q(t)=0,
$$

where the dots stand for time-derivatives, $\omega^{2}=c^{2} / \lambda_{L}^{2}$ is the frequency and $c=1 / \sqrt{\mu \varepsilon}$ is the velocity of the light in the superconductor. The expression (11) represents the equation of motion of a standard damped harmonic oscillator whose solution is

$$
q(t)=A e^{-\sigma t / 2 \varepsilon} \sin (\Omega t+\delta)
$$

where $A$ and $\delta$ are constants to be determined by the initial conditions and $\Omega$ is given by

$$
\Omega^{2}=\omega^{2}-\left(\frac{\sigma}{2 \varepsilon}\right)^{2},
$$

with $\Omega^{2}>0$ (oscillatory solutions). The Eq. (11) can be easily derived from the time-dependent classical Hamiltonian

$$
H(t)=e^{-\sigma t / \varepsilon} \frac{\Phi^{2}}{2 \varepsilon}+\frac{1}{2} e^{\sigma t / \varepsilon} \varepsilon \omega^{2} q^{2},
$$

where $q$ and $\Phi$ are canonical variables with $\Phi$ being the magnetic flux. This Hamiltonian is the well-known CaldirolaKanai Hamiltonian, which has been used in the literature to study time-dependent systems in various areas of physics $[19,30-35]$. It is also easily verified that for this case, the classical magnetic flux is given by

$$
\Phi=\varepsilon e^{\sigma t / \varepsilon} \dot{q}
$$

which can be rewritten as

$$
\Phi=L^{\prime}(t) i
$$

where $i=\dot{q}$ is the current within a certain volume of the superconductor and

$$
L^{\prime}(t)=\varepsilon e^{\sigma t / \varepsilon},
$$

represents the inductance of the London superconductor. What is more, Eq. (15) yields

$$
\epsilon=-\frac{d \Phi}{d t}=\varepsilon e^{\sigma t / \varepsilon} \omega^{2}(t) q=L^{\prime}(t) \omega^{2} q,
$$

which is the Faraday's law for the London superconductor. Therefore, the above results give a complete classical description of the London superconductor.

\subsection{Time-dependent mesoscopic LC Circuit}

In the present subsection, we are interested in studying the classical behavior of the time-dependent mesoscopic LC circuit. The classical scheme of this circuit is well-known. In the present case, it consists of an inductance $L(t)$ and a capacitance $C(t)$. The time-dependent capacitance enters the total voltage as $q_{1} / C(t)$, and the time-dependent inductance induces a magnetic field with the flux $\Phi_{1}=L(t) i_{1}$, 
which contributes to the voltage, that is, Faraday's law. Here, $i_{1}=\dot{q}_{1}$ is the current in the circuit. Then, by using Kirchhoff's laws, one readily obtains the equation of motion for the charge $q_{1}$ as

$$
\ddot{q}_{1}(t)+\frac{\dot{L}}{L} \dot{q}_{1}(t)+\omega_{1}^{2} q_{1}(t)=0,
$$

where $\omega_{1}^{2}=1 / L C$ is the resonant frequency of the circuit. In the follows, we assume that $L(t)$ and $C(t)$ vary exponentially with time at a constant rate in the form $[20,36]$

$$
L(t)=L_{0} e^{\eta t} \quad \text { and } \quad C(t)=C_{0} e^{-\eta t},
$$

where $\eta$ is a positive constant and $L_{0}=L(0)$ and $C_{0}=$ $C(0)$. So, we can rewrite the equation (19) as

$$
\ddot{q}_{1}(t)+\eta \dot{q}_{1}(t)+\omega_{0}^{2} q_{1}(t)=0,
$$

where $\omega_{0}^{2}=1 / L_{0} C_{0}$. At this point, we observe that the timedependence of the inductance gives rise to a damping $\eta$ in the amplitude of the system. Hence, $\dot{L}(t)$ behaves as an effective conductivity for the circuit (compare Eqs. (11) and (19)).

The equation of motion (21) can be derived from the Hamiltonian

$$
H_{1}(t)=e^{-\eta t} \frac{\Phi_{1}^{2}}{2 L_{0}}+\frac{1}{2} e^{\eta t} L_{0} \omega_{0}^{2} q_{1}^{2},
$$

where $\Phi_{1}$ is the magnetic flux which is given by

$$
\Phi_{1}=L_{0} e^{\eta t} \dot{q}_{1}=L(t) i_{1}
$$

Here, we note that the Hamiltonian (22) is similar to the Hamiltonian (14). From Eq. (23) we get Faraday's law as

$$
\epsilon_{1}=-\frac{d \Phi_{1}}{d t}=L_{0} e^{\eta t} \omega_{0}^{2} q_{1}=L(t) \omega_{0}^{2} q_{1}
$$

Furthermore, the solution of Eq. (21) is given by

$$
q_{1}(t)=B e^{-\eta t / 2} \sin \left(\Omega_{1} t+\xi\right),
$$

where the constants $B$ and $\xi$ are determined by the initial conditions and $\Omega_{1}^{2}>0$ is the modified frequency of the circuit, which is given by

$$
\Omega_{1}^{2}=\omega_{0}^{2}-(\eta / 2)^{2}
$$

At this stage, we let us observe that the mathematical formalism for describing the classical behavior of the London superconductor and the time-varying mesoscopic LC circuit with inductance and capacitance modulated exponentially at a constant rate is identical. In both cases, the equations of motion are governed by a standard damped harmonic oscillator [Eqs. (11) and (21)] which can be obtained from similar Hamiltonians [Eqs. (14) and (22)]. These systems also possess similar expressions for the magnetic flux [Eqs. (16) and (23)], inductance [Eqs. (17) and (20)] and Faraday's law [Eqs. (18) and (24)]. In order to push this analogy even further, we make the following correspondence: $\sigma / \varepsilon \Leftrightarrow \eta$ and $\varepsilon$
$\Leftrightarrow L_{0}$. However, the equivalence of these two systems is not complete. In fact, the dispersion relations $\omega$ and $\omega_{0}$, which are inherent to each physical system, differ, and as a consequence, the behavior of the electromagnetic oscillations of each system is different. On the other hand, in the absence of dissipation, that is, $\sigma=0$ and $\eta=0$, the Hamiltonians (14) and (22) reduce to that of a time-independent harmonic oscillator with $\varepsilon$ and $L_{0}$ playing the role of the mass of the standard time-independent mechanical oscillator.

\section{Quantum description}

In order to obtain the quantum description of our timedependent mesoscopic LC circuit or, equivalently, of the London superconductor, we must solve the time-dependent Schrödinger equation associated with the Hamiltonian (22) or Hamiltonian (14), respectively. Here, we consider the Hamiltonian (22). The time-dependent Schrödinger equation associated with this Hamiltonian is

$$
H_{1}|\Psi, t\rangle=i \hbar \frac{\partial}{\partial t}|\Psi, t\rangle,
$$

where the charge $q_{1}$ and the magnetic flux $\Phi_{1}$ now are canonical operators satisfying the commutation relation $\left[q_{1}, \Phi_{1}\right]=$ $i \hbar$ with $\Phi_{1}=-i \hbar \partial / \partial q_{1}$. The solutions of Eq.(27) can be obtained with the aid of the dynamic invariant method introduced by Lewis and Riesenfeld [26]. According to this method, if there exist a time-dependent nontrivial Hermitian invariant operator $I(t)$ (constant of motion) for the system described by the Hamiltonian (22), and it obeys the equation

$$
\frac{d I}{d t}=\frac{1}{i \hbar}\left[I, H_{1}\right]+\frac{\partial I}{\partial t}=0
$$

the solutions of the time-dependent Schrödinger equation (27) can be written in terms of orthonormalized eigenstates $\left|\phi_{n}, t\right\rangle$ of $I(t)$,

$$
\left.\left.I(t) \mid \phi_{n}, t\right)\right\rangle=\lambda_{n}\left|\phi_{n}, t\right\rangle,
$$

and phase functions $\beta_{n}(t)$ as

$$
\left|\psi_{n}, t\right\rangle=e^{i \beta_{n}(t)}\left|\phi_{n}, t\right\rangle,
$$

where the $\lambda_{n}$ are time-independent eigenvalues and the phase functions $\beta_{n}(t)$ are derived from the equation

$$
\hbar \frac{d \beta_{n}(t)}{d t}=\left\langle\phi_{n}, t\left|i \hbar \frac{\partial}{\partial t}-H_{1}(t)\right| \phi_{n}, t\right\rangle .
$$

with the orthonormality condition $<\phi_{n^{\prime}}, t \mid \phi_{n}, t>=\delta_{n^{\prime} n}$. In this paper, we assume a quadratic invariant in the form $[19,32,36]$

$$
I(t)=\frac{1}{2}\left[\left(\frac{q_{1}}{\rho}\right)^{2}+\left(\rho \Phi_{1}-L \dot{\rho} q_{1}\right)^{2}\right],
$$


where $\rho(t)$ is a time-dependent real function satisfying the Milne-Pinney equation [19,27, 28]

$$
\ddot{\rho}(t)+\eta \dot{\rho}(t)+\omega_{0}^{2} \rho(t)=\frac{1}{L^{2}(t) \rho^{3}(t)},
$$

with $L(t)$ given by equation (20).

Next, our task is to solve the eigenvalue Eq. (29). In order to do this, we will use the Fock state base since, as it is wellknown, the quantum behavior of some quantum systems, in particular quantum harmonic oscillator-type systems, is more obvious in Fock states, which are states with specific numbers of energy quanta. Then, let us introduce annihilation and creation-type operators $a(t)$ and $a^{\dagger}(t)$ defined by [19,26,37]

$$
\begin{aligned}
a(t) & =\left(\frac{1}{2 \hbar}\right)^{1 / 2}\left[\frac{q_{1}}{\rho}+i\left(\rho \Phi_{1}-L \dot{\rho} q_{1}\right)\right], \\
a^{\dagger}(t) & =\left(\frac{1}{2 \hbar}\right)^{1 / 2}\left[\frac{q_{1}}{\rho}-i\left(\rho \Phi_{1}-L \dot{\rho} q_{1}\right)\right],
\end{aligned}
$$

with

$$
\left[a(t), a^{\dagger}(t)\right]=1 \text {. }
$$

In terms of these operators, the invariant (32) can be rewritten as

$$
\left.I(t)=\hbar\left[a^{\dagger}(t) a(t)+\frac{1}{2}\right)\right] .
$$

From the Eqs. (36) and (37) we see that the eigenvalue equation for $I(t)$ (see Eq. (29)) can also be solved exactly, just as for the harmonic oscillator in the time-independent case by using the Fock states $|n, t\rangle$. So, defining the Hermitian number operator by $N=a^{\dagger} a$ so that $N|n, t\rangle=n|n, t\rangle$, we find that

$$
\begin{aligned}
I(t) & =\hbar\left(N+\frac{1}{2}\right) . \\
I(t)|n, t\rangle & =\hbar\left(n+\frac{1}{2}\right)|n, t\rangle, \\
a(t)|n, t\rangle & =n^{1 / 2}|n-1, t\rangle, \\
a^{\dagger}|n, t\rangle & =(n+1)^{1 / 2}|n+1, t\rangle .
\end{aligned}
$$

From Eq. (38) we see that the eigenstates of $I(t)$ are also eigenstates of $N$ and vice versa.

In what follows, we want to find the phase functions given by Eq. (31). By making the change $\left|\phi_{n}, t\right\rangle \rightarrow|n, t\rangle$ and after performing some basic calculations, we get that

$$
\beta_{n}(t)=-\left(n+\frac{1}{2}\right) \int_{0}^{t} \frac{1}{L(\tau) \rho^{2}(\tau)} d \tau .
$$

We now consider a particular solution of the Milne-Pinney Eq. (33) given by $[19,32]$

$$
\rho(t)=\frac{e^{-\eta t / 2}}{\left(L_{0} \Omega_{1}\right)^{1 / 2}} .
$$

For this case, Eq. (42) reduces to

$$
\beta_{n}(t)=-\Omega_{1}\left(n+\frac{1}{2}\right) t .
$$

Therefore, we can write the solutions of the Schrödinger Eq. (27) as

$$
\left|\psi_{n}, t\right\rangle=e^{i \beta_{n}(t)}|n, t\rangle,
$$

with $\beta_{n}(t)$ given by Eq. (44). The general solution to the Schrödinger Eq. (27) can be written as $|\Psi, t\rangle=$ $\sum_{n} c_{n}\left|\psi_{n}, t\right\rangle$, where the coefficients $c_{n}$ are constant.

Next, we use the Fock states to calculate some quantum properties for the quantized mesoscopic $L C$ circuit. To this end, we use the Eqs. (39), (40), and (41). After a little algebra, we find that

$$
\begin{aligned}
\langle I\rangle & =\hbar\left(n+\frac{1}{2}\right) \\
\left\langle q_{1}\right\rangle & =\left\langle\Phi_{1}\right\rangle=0 \\
\left\langle q_{1}^{2}\right\rangle & =\hbar \rho^{2}\left(n+\frac{1}{2}\right) \\
\left\langle\Phi_{1}^{2}\right\rangle & =\hbar\left[\frac{1}{\rho^{2}}+(L \dot{\rho})^{2}\right]\left(n+\frac{1}{2}\right) .
\end{aligned}
$$

By using the above expressions, we find the quantum variances as

$$
\begin{aligned}
\left(\Delta q_{1}\right)^{2} & =\left\langle q_{1}^{2}\right\rangle-\left\langle q_{1}\right\rangle^{2}=\hbar \rho^{2}\left(n+\frac{1}{2}\right) \\
\left(\Delta \Phi_{1}\right)^{2} & =\left\langle\Phi_{1}^{2}\right\rangle-\left\langle\Phi_{1}\right\rangle^{2} \\
& =\hbar\left[\frac{1}{\rho^{2}}+(L \dot{\rho})^{2}\right]\left(n+\frac{1}{2}\right) .
\end{aligned}
$$

Multiplication of (50) by (51) yields

$$
(\Delta q)(\Delta \Phi)=\hbar\left[1+(L \rho \dot{\rho})^{2}\right]^{1 / 2}\left(n+\frac{1}{2}\right),
$$

which represents the uncertainty principle for our system. By using the particular solution (43), the uncertainty principle (52) reduces to

$$
\left(\Delta q_{1}\right)\left(\Delta \Phi_{1}\right)=\frac{\hbar \omega_{0}}{\Omega_{1}}\left(n+\frac{1}{2}\right) .
$$

From (53), we see that the uncertainty principle does not depend on time and that its value becomes larger when the dissipation, that is, $\eta$ increases. What is more, the above results give us the quantization of the charge, the magnetic flux, and the uncertainty principle for our time-dependent mesoscopic LC circuit. Now it is worth noticing that in the absence of the dissipation, $\eta=0$, the expression (53) becomes

$$
\left(\Delta q_{1}\right)\left(\Delta \Phi_{1}\right)=\hbar\left(n+\frac{1}{2}\right)
$$


which is similar to the uncertainty principle of a harmonic oscillator with frequency $\omega_{0}$ (see Eq. (21)). We also notice that for this case, the particular solution (43) becomes $\rho=1 /\left(L_{0} \omega_{0}\right)^{1 / 2}$ and the Hamiltonian (22) and the annihilation and creation operators (35) and (36), respectively, are formally reduced to that of the standard time-independent harmonic oscillator.

Finally, we observe that for the London superconductor, the quantum description can be carried out following the same procedure used for the time-dependent nanoscale LC circuit case since, as we have demonstrated previously, the mathematical framework to study the behavior of both systems is identical.

\section{Summary}

In this work, we have presented a simple procedure to analyze the classical and quantum behavior of the London superconductor and of a mesoscopic LC circuit with an inductance and a capacitance varying exponentially with time at a constant rate. Notably, we have found that the behavior of these two systems is equivalent, both classically and quantum mechanically. We also have shown that this behavior can be mapped into, in both cases, a standard damped harmonic oscillator which is governed by the well-known CaldirolaKanai Hamiltonian. Yet, we have noted that $\dot{L}(t)$ behaves as an effective conductivity for the mesoscopic $L C$ circuit. Further, by using the invariant method, appropriated annihilation, and creation-type operators and Fock states, we have easily solved the time-dependent Schrödinger equation for the time-dependent mesoscopic LC circuit (the same procedure can be made for the London superconductor) and written its solutions in terms a particular solution of the nonlinear Milne-Pinney equation. In addition, by using Fock states, we have calculated the expectation values of the charge and magnetic flux, their quantum variances as well as the uncertainty principle for the quantized time-dependent mesoscopic LC circuit. Finally, we expect that the procedure developed in this paper can be helpful to investigate subjects related to London superconductors and other mesoscopic or nanoscale circuits.
1. X. G. Meng, J. S. Wang and B. L. Liang, Quantum theory of a mutual-inductance-coupled LC circuit including Josephson junctions studied via the entangled state representation, Solid State Commun. 149 (2009) 2027 https://doi.org/10. $1016 / j . s s c .2009 .08 .028$

2. J. S. Wang, B. L. Liang and H. Y. Fan, Operator Josephson equation for a Josephson junction connected into a mesoscopic LC circuit, Mod. Phys. Lett. B 22 (2008) 3171, https : //doi.org/10.1142/S0217984908017692

3. L. M. K. Vandersypen et al, Experimental realization of Shor's quantum factoring algorithm using nuclear magnetic resonance, Nature 414 (2001) 883, https://doi.org/10.1038/ $414883 a$

4. S. Gulde et al., Implementation of the Deutsch-Jozsa algorithm on an ion-trap quantum computer, Nature 421 (2003) 48, https://doi.org/10.1038/nature01336

5. Q. A. Turchette, et al., Measurement of Conditional Phase Shifts for Quantum Logic, Phys. Rev. Lett. 75 (1995) 4710, https://doi.org/10.1103/PhysRevLett.75. 4710

6. X. G. Meng, J. S. Wang and B. L. Liang, Modified Josephson equations for the mesoscopic LC circuit including two coupled Josephson juctions, J. Phys. A: Math. Theo.41 (2008) 235208, https://doi.org/10.1088/ 1751-8113/41/23/235208

7. H. K. Onnes, Further experiments with liquid helium. H. On the electrical resistance of pure metals etc. VII. The potential difference necessary for the electric current through mercury below $4^{\circ}-19$ K., Commum. Phys. Lab. Univ., Leiden 18a (1911) 273, https://doi.org/10.1007/ 978-94-009-2079-8_18
8. W. Meissner and R. Ochsenfeld, Ein neuer Effekt bei Eintritt der Supraleitfähigkeit, Naturwiss. 21 (1933) 783, https: //doi.org/10.1007/BF01504252

9. F. London and H. London, The Electromagnetic Equations of the Supraconductor, Proc. Roy. Soc. Lond. A149, (1935), 71, http://doi.org/10.1098/rspa.1935.0048

10. V. L. Ginzburg and L. D. Landau, On the theory of superconductivity, In: On Superconductivity and Superfluidity. Springer, Berlin, Heidelberg. https://doi.org/10. 1007/978-3-540-68008-6_4

11. J. Bardeen, L. N. Cooper and J. R. Schrieffer, Theory of Superconductivity, Phys. Rev. 108, (1957), 1175, https://doi. org/10.1103/PhysRev.108.1175

12. B. D. Josephson, Possible new effects in superconductive tunnelling, Phys. Lett. 1 (1962) 251, https://doi .org/10. 1016/0031-9163(62) 91369-0

13. F. London, Superfluids, (Dover Publications, INC., New York, 2nd ed, 1961), pp. 27-91

14. I. A. Pedrosa, Quantum description of a time-dependent mesoscopic RLC circuit, Phys. Scr. T151 (2012) 014042, https://doi.org/10.1088/0031-8949/2012/ T151/014042

15. M. A. Kastner, The single-electron transistor, Rev. Mod. Phys. 64 (1992) 849, https://doi.org/10.1103/ RevModPhys.64.849

16. J. R. Choi, Quantization of underdamped, critically damped, and overdamped electric circuits with a power source Int. J. Theo. Phys. 41 (2002) 1931, https://doi.org/10. 1023/A: 1021056924059

17. J. R. Choi, Exact solution of a quantized LC circuit coupled to a power source, Phys. Scr. 73 (2006) 587, https://doi. org/10.1088/0031-8949/73/6/010 
18. B.L. Liang, J.S. Wang and X.G. Meng, Quantization for the mesoscopic RLC circuit and its thermal effect by virtue of GHFT, Mod. Phys. Lett. B 23 (2009) 3621, https : //doi. org/10.1142/S0217984909021661

19. I. A. Pedrosa and A.P. Pinheiro, Quantum Description of a Mesoscopic RLC Circuit, Prog. Theo. Phys. 125 (2011) 1133, https://doi.org/10.1143/PTP.125.1133

20. I. A. Pedrosa, J. L. Melo and S. Salatiel, Quantization, coherent states and geometric phases of a generalized nonstationary mesoscopic RLC circuit, Eur. Phys. J. B 87 (2014) 269, https://doi.org/10.1140/epjb/ e2014-50174-5

21. W. H. Louisell, Quantum Statistical Properties of Radiation, (John Wiley, New York, 1st ed, 1973),pp. 230-235

22. H. M. Luo, L. and X. Q. Ke, Thermodynamics of squeezed state for mesoscopic RLC circuits, Int. J. Theor. Phys. 46 (2007) 2935, https://doi.org/10.1007/ s10773-007-9406-2

23. D. Xu, Hannay angle in an LCR circuit with time-dependent inductance, capacity and resistance, J. Phys. A: Math. Gen. 35 (2002) L455, https://doi.org/10.1088/ 0305-4470/35/29/104

24. L. F. Wei and X. L. Lei, Dynamics for a Mesoscopic RLC Circuit with a SourcePhys. Scripta 62 (2000) 7, https : / / doi. org/10.1238/Physica.Regular.062a00007

25. Z. M. Zhang, L. S. He and S. K. Zhou, A quantum theory of an RLC circuit with a source, Phys. Lett. A 244 (1998) 196, https://doi.org/10.1016/S0375-9601(98) 00295-3

26. H. R. Lewis Jr. and W. B. Riesenfeld, An exact quantum theory of the time-dependent harmonic oscillator and of a charged particle in a time-dependent electromagnetic field, J. Math. Phys. (N.Y.) 10 (1969) 1458, https://doi.org/10.1063/1. 1664991

27. W. E. Milne, The numerical determination of characteristic numbers, Phys. Rev. D 35 (1930) 863, https : / doi .org/ $10.1103 /$ PhysRev. 35.863
28. W. K. Schief, A discrete Pinney equation, Appl. Math. Lett. 10 (1997) 13, https://doi.org/10.1016/ S0893-9659(97)00026-8

29. V. Aguiar et al, London superconductivity approach in a time-dependent background, Physica C 580 (2021) 1353783, https://doi.org/10.1016/j.physc.2020. 1353783

30. P. Caldirola, Quantum theory of nonconservative systems, IL Nuovo Cimento, 77 (1941) 241, https://doi.org/10. $1007 / \mathrm{BF} 02721487$

31. E. Kanai, On the quantization of the dissipative systems, Prog. Theor. Phys. 3 (1948) 440, https: //doi.org/10.1143/ $\mathrm{ptp} / 3.4 .440$

32. I. A. Pedrosa, G. P. Serra and I. Guedes, Wave functions of a time-dependent harmonic oscillator with and without a singular perturbation, Phys. Rev. A, 56 (1997) 4300, https: //doi.org/10.1103/PhysRevA.56.4300

33. R. W. Hasse, On the quantum mechanical treatment of dissipative systems, J. Math. Phys. 16 (1975) 2005, https: //doi.org/10.1063/1.522431

34. J. R. Choi, Coherent and squeezed states for light in homogeneous conducting linear media by an invariant operator method, Int. J. Theo. Phys. 43 (2004) 2113, https : / / doi .org/10 . 1023/B:IJTP.0000049014.09671.e2

35. J. R. Choi, Quantum description of the electromagnetic waves in homogeneous conducting linear media, J. Opt. B 5 (2003) 409, https://doi.org/10.1088/1464-4266/5/5/ 006

36. I. A. Pedrosa, J. L. Melo and E. Nogueira Jr., Linear invariants and the quantum dynamics of a nonstationary mesoscopic RLC circuit with a source, Mod. Phys. Lett. B 28 (2014) 1450212, https://doi.org/10.1142/S0217984914502121

37. I. A. Pedrosa, Quantum electromagnetic waves in nonstationary linear media, Phys. Rev. A 83 (2011) 032108, https: //doi.org/10.1103/PhysRevA.83.032108 